\title{
HPCSA Serious Injury Narrative Test guideline
}

H J Edeling, MB BCh, FCS (SA) (Neuro), CIME (ABIME); N B Mabuya, MB BCh, MBA, Dip (Occ Med), BSc Hons (Aer Med), CIME (ABIME); P Engelbrecht, MB ChB, MMed (Ort), CIME (ABIME); K D Rosman, MB ChB, MMed (Neuro), MD, FC (Neurol) (SA), CIME (ABIME); D A Birrell, MB ChB, FRCS (Edin)

Health Professions Council of South Africa Appeal Tribunals, Pretoria, South Africa

This guideline is published by the Health Professions Council of South Africa Appeal Tribunals to define the use of a Serious Injury Narrative Test Report, as well as the required structure, content and criteria thereof.

Corresponding author: H J Edeling (edeling@emlct.com)

Current South African Road Accident Fund (RAF) legislation requires a medical determination of the seriousness of injuries sustained in motor vehicle accidents to determine whether the claimant is entitled to a claim for general damages. Such medical assessments are submitted in the form of RAF 4 Serious Injury Assessment Reports. Contested claims for serious injury are referred to the Health Professions Council of South Africa (HPCSA) Appeal Tribunals for final determination. The legislation prescribes 2 instruments, namely the American Medical Association (AMA) Guides (6th edition) and the Narrative Test for this purpose. Whereas the AMA Guides are published in a comprehensive book, and training courses are provided in their use, existing legislation does not provide any indication of the required structure, content or criteria of a Narrative Test report. This document is published by the HPCSA Appeal Tribunals as a guideline to the performance of the Narrative Test; what it is, reasons for applying it and who should compile it, as well as the required structure, content and criteria thereof. A Narrative Test Report should include relevant and meaningful comment in relation to each of the 6 sections described in the article.

S Afr Med J 2013;103(10):763-766. DOI:10.7196/SAMJ.7118

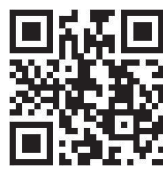

\section{Summary}

This guideline is an aide memoire to medical practitioners and other relevant experts compiling Narrative Test Reports. A Narrative Test Report should include relevant and meaningful comment for each of the following:

1.1 Injury diagnosis/nature and extent of injuries in the acute post-traumatic period

- Diagnosis by medical practitioner.

- Opinion of medical practitioner re nexus between the accident and diagnosed injuries.

1.2 Outcome diagnosis/nature and extent of permanent impairment after maximal medical improvement

- Diagnosis by medical practitioner.

- Opinion of medical practitioner re nexus between the accident and diagnosed injuries.

1.3 External/environmental/contextual circumstances of the person's life - either altered or unaltered

- Factual description by medical practitioner and/or other relevant expert(s).

- Opinion of medical practitioner re nexus between injuries sustained in the accident and any changes in external circumstances.

1.4 Individual circumstances of the person's life either altered or unaltered, including functional impairment

- Factual description by medical practitioner and/or other relevant experts.

- Opinion of medical practitioner re nexus between injuries sustained in the accident and any changes in individual circumstances.
1.5 Chronic pain, subjective suffering and/or loss of enjoyment of life

- Factual description by medical practitioner and/or other relevant experts.

- Opinion of medical practitioner and/or other relevant experts in relation to the credibility, congruence and consistency or otherwise of the complaints.

- Opinion of medical practitioner re nexus between injuries sustained in the accident and reported subjective suffering.

\subsection{Level or degree of changes}

Comment by medical practitioner and/or other relevant experts, utilising meaningful semi-quantitative terminology, e.g. insignificant, trivial, inconsequential, mild, moderate, severe, intrusive, overwhelming, devastating, significant.

\section{The Narrative Test}

\subsection{What is the Narrative Test?}

The Narrative Test is a medical instrument prescribed by the Road Accident Fund (RAF) Amendment Regulations, 2008 ${ }^{[1]}$ to the RAF Amendment Act, 2005, ${ }^{[2]}$ which amends the RAF Act $56,1996 .{ }^{[3]}$

The Narrative Test stands apart from the American Medical Association (AMA) 'Guides to the Evaluation of Permanent Impairment ${ }^{\left[{ }^{[4]}\right.}$ and cannot be defined or interpreted in terms of these.

The RAF Amendment Regulations do not provide any guidelines to the structure, content or criteria of the Narrative Test. This guideline is published by the HPCSA Appeal Tribunals as a guideline to the performance of the Narrative Test, as well as the required structure, content and criteria thereof. 


\subsection{Reasons for applying the Narrative Test}

The need for the Narrative Test arises in any case where:

- The injuries are found to have resulted in $<30 \%$ whole person impairment (WPI) according to the method of the AMA Guides; and

- The medical practitioner who is drafting the RAF 4 Serious Injury Assessment Report nonetheless regards the injuries as serious.

There are two reasons for cases that have been regarded as serious by HPCSA Appeal Tribunals despite having $<30 \%$ WPI according to the method of the AMA Guides:

- The failure of the AMA Guides to take the 'circumstances of the third party' into account properly or effectively. ${ }^{\left[{ }^{[}\right]}$

- Inherent shortcomings of the AMA Guides, especially with respect to estimating the life-altering impact of injuries that have resulted in more abstract and subjective impairments and suffering. ${ }^{[5]}$

The RAF Amendment Act ${ }^{[2]}$ stipulates in section 17(1A)(a) that the 'assessment of a serious injury shall be based on a prescribed method adopted after consultation with medical service providers and shall be reasonable in ensuring that injuries are assessed in relation to the circumstances of the third party'.

In highlighting the importance of the 'circumstances of the third party', the Act effectively prescribes an assessment of 'disability' as opposed to an assessment of 'impairment'.

In contrast to the requirements of the Act, the AMA Guides prescribe an impairment rating system, which for practical purposes excludes consideration of the 'circumstances of the third party'.

The AMA Guides define impairment and disability as follows:

- Impairment: 'a significant deviation, loss, or loss of use of any body structure or body functions in an individual with a health condition, disorder, or disease'.

- Disability: 'activity limitations and/or participation restrictions in an individual with a health condition, disorder, or disease'.

The AMA Guides do not provide for any assessment of the nature or degree of permanent disability. The AMA Guides ${ }^{[4]}$ state (page 6):

- 'The Guides is not intended to be used for direct estimates of work participation restrictions. Impairment percentages derived according to the Guides' criteria do not directly measure work participation restrictions.'

- 'In disability evaluation, the impairment rating is one of several determinants of disablement. Impairment rating is the determinant most amenable to physician assessment; it must be further integrated with contextual information typically provided by nonphysician sources regarding psychological, social, vocational, and avocational issues.'

\subsection{Who should compile a Narrative Test Report?}

The RAF Amendment Regulations ${ }^{[1]}$ stipulate that the RAF 4 Serious Injury Assessment Report, including the Narrative Test Report, should be compiled by a 'medical practitioner', defined as a medical practitioner registered in terms of the Health Professions Act, 1974. ${ }^{[6]}$

For a variety of reasons, although medical practitioners should be able to provide adequately detailed Narrative Test Reports in certain cases, it is found in practice that in many cases medical practitioners do not provide adequate factual descriptions of relevant or altered 'circumstances of the third party'.

It is, therefore, recommended that the Narrative Test Report provided by a medical practitioner should generally be supplemented by reports from other relevant experts, mainly to properly describe the relevant or altered 'circumstances of the third party'.

In this context, 'other relevant experts' refers principally to occupational therapists. Depending on the nature of the impairments and the particular 'circumstances of the third party', however, supplementary reports may be required of neuropsychologists, educational psychologists, speech therapists, and/or industrial psychologists.

In reference to the structure and content of a Narrative Test Report (see section 2.4):

- Sections 1 and 2 should be compiled by the medical practitioner.

- Sections 3 - 6 may be compiled by the medical practitioner or may be compiled in the supplementary report(s) of the other relevant expert(s) (see below); in which case comment should be provided by the medical practitioner (see below).

- The supplementary report of a relevant expert should refer to the diagnoses of the medical practitioner in Sections 1 and 2, and should deal in detail with Sections 3 - 6 .

- Where Sections 1 and 2 of the Narrative Test Report of the medical practitioner are not available to the other relevant expert(s) at the time of compiling their report, bearing in mind inter alia that the scope of practice of such relevant experts precludes the formulation of medical diagnoses, the other relevant expert(s) should refer to the injury diagnosis and outcome diagnosis of medical practitioners as documented in other available medical records or reports.

- Where available records or reports document only an injury diagnosis but not an outcome diagnosis, the other relevant expert(s) should, on the basis of their own observations and expertise, provide a working description of the impairments (equivalent to an outcome diagnosis) and defer to the medical practitioner for final formulation of the outcome diagnosis.

- Where Sections 3 - 6 have been compiled in the supplementary report of the other relevant expert(s), the medical practitioner should read the report of the other relevant expert(s), and should provide further comment in line with the requirements as set out below.

\subsection{The structure and content of a Narrative Test Report} A Narrative Test Report should include relevant and meaningful comment in relation to each of the following sections:

\subsubsection{Section 1: Injury diagnosis (acute)}

The diagnosis of injuries sustained in the accident should be recorded, i.e. a name describing each injury during the acute posttraumatic period.

The injury diagnosis/diagnoses should be formulated by a medical practitioner.

In addition, the medical practitioner should provide opinion in relation to the nexus between the accident and diagnosed injuries.

Examples of injury diagnoses are:

- compound fracture of the left femur

- head injury with severe traumatic brain injury

- soft tissue injury of the lumbar spine

- psychological trauma.

\subsubsection{Section 2: Outcome diagnosis (permanent)}

The diagnosis of the chronic condition that has arisen from the injuries should be recorded, i.e. a meaningful name describing each chronic post- 
traumatic condition following maximal medical improvement (MMI). For purposes of the Narrative Test, MMI is defined as 'a point at which the patient's condition is considered to have stabilised, and taking into account the medical and surgical treatment available to them, further recovery or deterioration is not anticipated over the following 12 months within medical probability'.

MMI does not preclude the deterioration of a condition that is expected to occur with the passage of time, or as a result of the normal ageing process or possible future complications, nor does it preclude allowances for ongoing follow-up for optimal maintenance of the medical condition in question.

The outcome diagnosis also serves as a description of permanent impairment following the accident.

The outcome diagnosis/diagnoses should be formulated by a medical practitioner.

In addition, the medical practitioner should provide opinion in relation to MMI, and in relation to the nexus between injury diagnosis and outcome diagnosis.

Examples of outcome diagnoses are:

- post-fracture syndrome with malunion and deformity

- post-traumatic organic brain syndrome

- intermittent mechanical back pain

- post-traumatic stress disorder.

\subsubsection{Section 3: External circumstances of the person's life}

A factual description should be recorded of the external circumstances of the person's life, i.e. the environmental or contextual circumstances.

These circumstances generally remain unaltered following the accident, but in case of any change such changes should be recorded.

External circumstances include:

- geographical location

- type of accommodation

- family support

- financial status

- cultural affiliation

- religious affiliation

- access to transport

- access to healthcare.

In terms of this section of the Narrative Test Report, it is acceptable and generally advisable for the medical practitioner to refer to the supplementary report(s) of other relevant experts (see section 2.3), in which case it is not necessary for the medical practitioner to duplicate such factual descriptions in their report.

It is, however, necessary for the medical practitioner to indicate that they have read such supplementary reports and to express an opinion in relation to the nexus between injuries sustained in the accident and any reported changes in external circumstances.

\subsubsection{Section 4: Individual circumstances of the person's life and functional impairment}

A factual description of pre-accident individual circumstances should be recorded, i.e. the personal circumstances that are more vulnerable to change or loss flowing from any permanent impairment.

This should be followed by factual descriptions of functional impairment after MMI, including altered and unaltered post-accident individual circumstances.

Changes in these individual circumstances typically describe the nature and elements of permanent disability.

Individual circumstances include:

- basic and advanced activities of daily living (conveniently set out in the AMA Guides, ${ }^{[4]}$ page 323 )
- personal amenities such as sporting and other recreational activities

- life roles such as parent, child, sibling, spouse, partner, friend, breadwinner, mentor, supervisor, caregiver, etc.

- independence or degree of dependency

- educational status and capacity

- employment status and capacity.

In terms of this section of the Narrative Test Report, it is acceptable and generally advisable for the medical practitioner to refer to the supplementary report(s) of other relevant experts (see section 2.3), in which case it is not necessary for the medical practitioner to duplicate such factual descriptions in their report.

It is, however, necessary for the medical practitioner to indicate that they have read such supplementary reports and to express an opinion in relation to the nexus between injuries sustained in the accident and findings of the other relevant expert(s) regarding functional impairment and altered post-accident individual circumstances.

\subsubsection{Section 5: Chronic pain, subjective suffering and/or loss of} enjoyment of life

The consequences of injuries and impairment that are referred to above are largely tangible and objectively determinable. Injuries and impairments may also result in variable degrees of subjective suffering that is more abstract and difficult to measure.

Bearing in mind that compensation for 'general damages' relates largely to compensation for 'pain, suffering and loss of enjoyment of life', all of which are both subjective and abstract, a proper assessment of subjective and abstract suffering is necessary.

A factual description of any accident-related pain, subjective suffering and/or loss of enjoyment of life should be recorded by the medical practitioner and/or other relevant experts.

Because such subjective sequelae of injuries are not amenable to objective or concrete measurement, and because their assessment is more difficult than that of more tangible/concrete sequelae, the report should include opinion based on mindful professional judgement by the medical practitioner and/or the other relevant other expert(s) in relation to the credibility, congruence and consistency or otherwise of the complaints.

In addition, the medical practitioner should provide opinion in relation to the nexus between injuries sustained in the accident and reported pain, suffering and/or loss of enjoyment of life.

\subsubsection{Section 6: Level/degree of changes}

The consequences of injuries, as seen in relation to the 'circumstances of the third party', essentially describe the nature and elements of permanent disability.

In addition to the nature and elements of permanent disability, determination of the seriousness of injuries requires an assessment of the level or degree of permanent disability, i.e. the level or degree of activity limitations, participation restrictions and subjective suffering.

The report should, therefore, include comment by the medical practitioner and/or the other relevant experts, based on reported facts as well as application of mindful professional judgement, in relation to the level or degree of activity limitations, participation restrictions and subjective suffering, i.e. the significance or otherwise of the changes to the life of the injured person.

Whereas it is not feasible to express such opinions in a rigid quantitative manner (e.g. a percentage rating of permanent disability), it is both feasible and necessary to express meaningful semi-quantitative opinions using terminology, e.g insignificant, trivial, inconsequential, mild, moderate, severe, intrusive, overwhelming, devastating, significant. 


\subsection{Criteria for assessment of serious injuries}

HPCSA Appeal Tribunals regard injuries as serious when it is evident that the injuries have resulted in 'significant life changing sequelae'.

When considering the significance of injury sequelae, the following should be regarded:

- the nature and elements of permanent disability (sections 2.4.2 2.4.5), and

- the level or degree of limitations, restrictions and subjective suffering (section 2.4.6).

For example:

- Chronic pain may be intermittent mild to moderate pain that occurs twice a month, is relieved by simple analgesics and does not interfere significantly with activities. This would not be regarded as serious.

- On the other hand, chronic pain that has been found by the medical practitioner to be congruent with established conditions as well as being credible and consistent, may be constant moderate to severe pain that is only partially relieved by compound or narcotic analgesics and that does interfere significantly with activities. This would be regarded as serious.

- The loss of employment capacity related to subtle mental impairment of an assembly line worker who has become dependent on some degree of structure and supervision in the workplace, but for whom such structure and supervision have always formed an integral part of the job, and who has remained in the same employment and continued to satisfy the requirements of the employer, would not be regarded as serious.
- On the other hand, the loss of employment capacity related to subtle mental impairment of an advocate who has lost the ability to succeed in Court as well as loss of enjoyment of life related to losses of professional standing, respect and independence would be regarded as serious.

Whereas it is not possible to provide a concretely measurable definition of 'significant life changing sequelae', experience at HPCSA Appeal Tribunal meetings shows that a panel of experienced medical practitioners who are provided with the sufficient relevant information (as set out above) are generally and readily able to reach consensus in relation to cases where injuries have resulted in 'significant life changing sequelae' and cases where injuries have not resulted in 'significant life changing sequelae.

Therefore, it is recommended that a determination of whether injuries have resulted in 'significant life changing sequelae' or not should be the final criterion for evaluation of injuries as serious or not serious by the Narrative Test.

\section{References}

1. The Road Accident Fund Regulations, 2008. Government Gazette no. 31249, notice no. 770, 21 July 2008. http://jaa.org.za/documents/docs/DOC210513-21052013064909.pdf (accessed 17 July 2013).

2. The Road Accident Fund Amendment Act 19, 2005. Government Gazette no. 28374, 5 January 2006. http://www.info.gov.za/view/DownloadFileAction?id=67871 (accessed 17 July 2013).

3. The Road Accident Fund Act 56 of 1996. Government Gazette no. 1756, 1 November 1996. http://www. info.gov.za/gazette/acts/1996/a56-96.htm (accessed 17 July 2013).

4. Rondinelli RD. Guides to the Evaluation of Permanent Impairment. 6th ed. Atlanta: AMA, 2008.

5. Slabbert M, Edeling HJ. The Road Accident Fund and serious injuries: The Narrative Test. . Slabbert M, Edeling HJ. The Road Accident Fund and serious injuries: The Narra
Potchefstroom Electronic Law Journal 2012;15(2). [http://dx.doi.org/10.4314/pelj.v15i2.10]

6. Health Professions Act No. 56, 1974. http://www.hpcsa.co.za/downloads/health_act/health_act_56_1974. 6. Health Professions Act No. 56
pdf (accessed 17 July 2013). 